\title{
ESTUDO DO COMPORTAMENTO DE EMULSÕES DO TIPO A/O
}

\author{
V. F. DORNELAS ${ }^{1}$, A. P. MENEGUELO ${ }^{1}$, D. C. RIBEIRO ${ }^{1}$ \\ ${ }^{1}$ Universidade Federal do Espírito Santo, Departamento de Engenharia e Tecnologias \\ E-mail para contato: vitoriafeliciodornelas@ hotmail.com
}

\begin{abstract}
RESUMO - Durante toda a cadeia produtiva o petróleo é exposto a agitação que, combinada com temperatura, pressão e produtos químicos presentes no óleo, resulta na formação de emulsões. Atualmente há uma demanda de estudos sobre emulsões, principalmente, devido a descoberta de reservas de petróleo pesado, que formam emulsões mais estáveis devido a maior presença de asfaltenos em sua composição. Este trabalho apresenta um estudo experimental e teórico sobre o comportamento de emulsões $\mathrm{A} / \mathrm{O}$ quanto aos parâmetros concentração de emulsificante, taxa de cisalhamento e fração volumétrica de água. Foram analisados parâmetros reológicos e de estabilidade das emulsões. Os resultados experimentais para a viscosidade medidos pelo viscosímetro foram comparados aos valores calculados através da curva de fluxo. Foi observado que os valores medidos pelo viscosímetro são suficientemente próximos aos valores calculados, comprovando assim a viscosidade de cada uma das emulsões e o seu comportamento Newtoniano.
\end{abstract}

\section{INTRODUÇÃO}

Uma emulsão é a mistura de dois ou mais líquidos que são normalmente imiscíveis. Macroemulsões, ou simplesmente emulsões, são dispersões de líquido em líquido, com tamanho de gota tipicamente variando de 1 a 100 micrometros (HENRÍQUEZ, 2009).

Nesta faixa, as gotas da emulsão são em geral grandes o suficiente para sedimentar através da influência gravitacional. Estes sistemas possuem uma estabilidade mínima, podendo melhorar com a adição de agentes ativos de superfície. Geralmente, emulsões são estabilizadas por um agente surfactante que têm duas funções básicas: diminuir a tensão interfacial, favorecendo a formação de emulsão; e evitar a coalescência entre as gotas uma vez obtida a emulsão (HENRÍQUEZ, 2009).

$\mathrm{Na}$ indústria do petróleo emulsões de água em óleo estão presentes nas operações de produção e no setor de refino. Na produção o teor de água pode chegar até $60 \%$ em volume. Emulsões são formadas por misturas de água e óleo que escoam em regime turbulento ou devido ao efeito do gradiente de pressão em poros do reservatório, chokes, ou em válvulas utilizadas nas tubulações. São, em geral, fluidos de comportamento não-Newtoniano, com viscosidade dependente da fração volumétrica da fase dispersa, da temperatura e principalmente da taxa de cisalhamento. Outros fatores, como tamanho da gota, distribuição do tamanho das gotas, a viscosidade e a densidade do óleo também influenciam no comportamento da emulsão. 
Além da alta viscosidade associada aos óleo pesados, a formação de emulsões (A/O) durante o escoamento contribui ainda mais para o aumento da perda de carga e redução da produção. Portanto é importante estudar maneiras de reduzir a viscosidade de óleos pesados para garantir seu escoamento. As pesquisas em emulsão inversa na produção de petróleo visam diminuir a viscosidade do óleo pesado, para que as perdas energéticas sejam as menores possíveis e que seja viabilizada extração do óleo cru (SOUZA, 2009).

\subsection{Classificação das Emulsões}

Emulsões produzidas em campo de produção de petróleo podem ser classificadas em três grupos: água em óleo (A/O), Figura 1-1, óleo em água (O/A), Figura 1-2, e emulsões múltiplas ou complexas (A/O/A ou O/A/O), Figuras 1-3 e 1-4 (LAKE, 2006).

Figura 1 - Representação esquemática das estruturas das emulsões simples e múltiplas. Em (1) emulsão A/O, (2) O/A, (3) A/O/A e (4) O/A/O. (PEREIRA \& GRACIA-ROJAS, 2015).

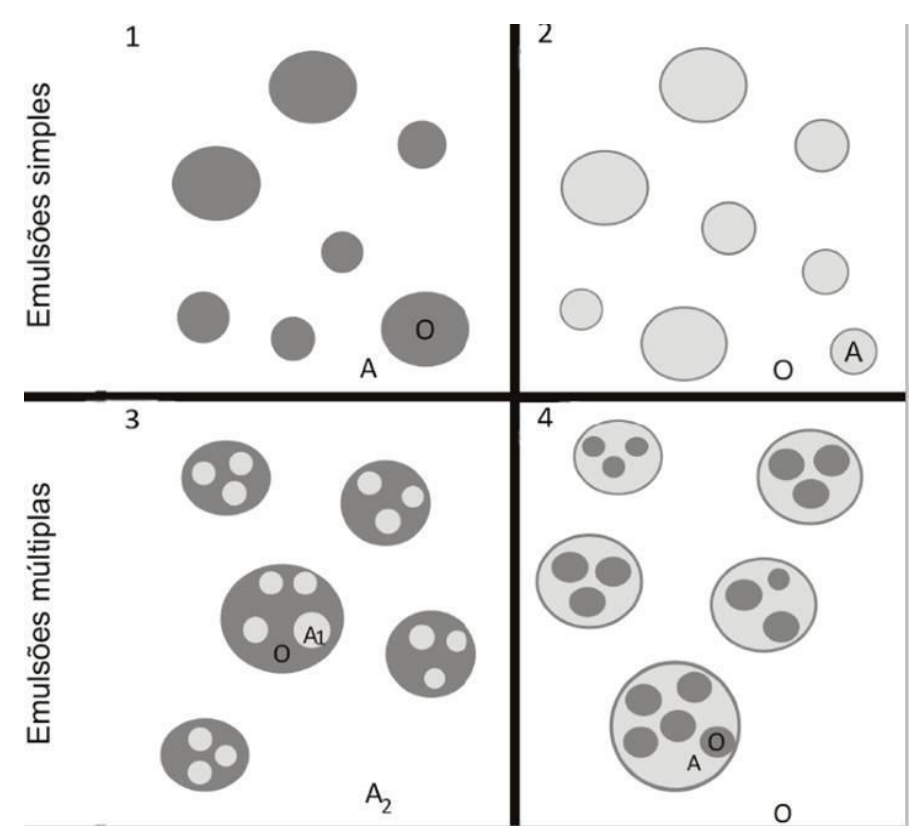

As emulsões podem ser classificadas quanto ao seu grau de estabilidade, sendo consideradas fracas quando há separação de água livre em poucos minutos, médias quando a separação de fase ocorre em 10 minutos e fortes quando a separação de fases leva algumas horas ou dias (SANTANA, 2013).

\subsection{Tipos de Fluidos}

Os fluidos podem ser caracterizados quanto ao seu comportamento reológico, sendo que os fluidos mais comuns são facilmente identificados pela análise do perfil das curvas de fluxo 


\section{Congresso Brasileiro de Engenharia Química em Iniciação Científica UFSCar - São Carlos - SP 16 a 19 de Julho de 2017}

e/ou viscosidade. Os principais tipos de fluidos estão relacionados na Figura 2 (NASCIMENTO, 2008)

Figura 2 - Curva de fluxo e curva de viscosidade (NASCIMENTO, 2008).

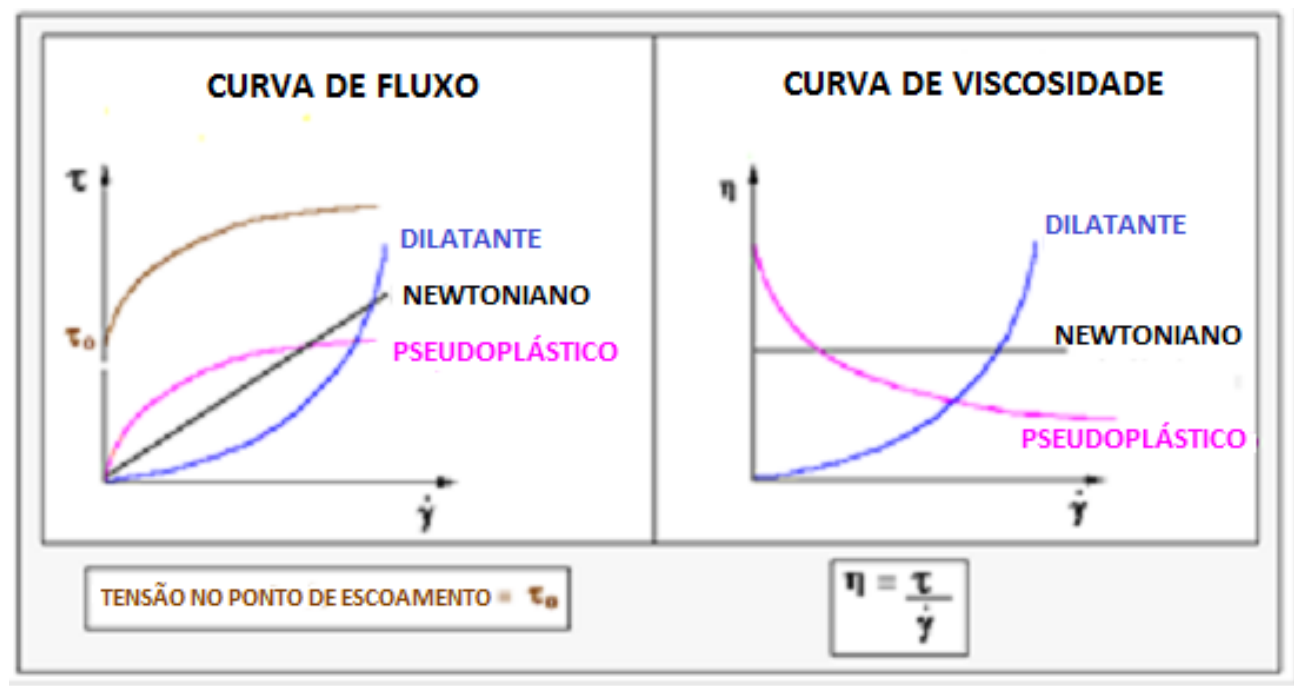

O comportamento Newtoniano é característico de emulsões bem diluídas, e nesse caso, a viscosidade do fluido independe da taxa de cisalhamento. Os primeiros estudos sobre viscoplasticidade realizados por Bingham em 1992 mostraram que existe um valor residual para a tensão de cisalhamento - tensão limite de escoamento - que precisa ser excedido para que o material apresente fluxo viscoso (Navarro, 1997 apud HERMANY, 2012). Fluidos dilatantes são caracterizados pelo aumento da sua viscosidade com o aumento da taxa de cisalhamento, já fluidos pseudoplásticos são caracterizados pela diminuição da viscosidade com o aumento da taxa de cisalhamento.

\section{METODOLOGIA}

Para realizar o estudo do comportamento das emulsões $\mathrm{A} / \mathrm{O}$ foi definido um procedimento experimental para a etapa de preparo e caracterização das emulsões formadas. Todas as emulsões foram preparadas com o auxílio de um homogeneizador mecânico à 8000 RPM por 5 minutos. Os fluidos utilizados para o preparo da emulsão foram: água destilada, óleo diesel e o surfactante SPAN 80.

Para análise do comportamento e caracterização da emulsão foi analisado a influência do teor de água. As seguintes proporções de água foram utilizadas: 5, 10, 20, 30, 40, 50, 60 e $70 \%$ foram adicionados $0,25 \%$ de SPAN 80 em cada uma das amostras. A Tabela 1 apresenta a quantidade em volume da composição de cada uma das emulsões. 
Tabela 1 - Proporção em volume dos componentes da emulsão.

\begin{tabular}{c|ccc} 
Emulsão & Óleo Diesel (\%) & Água (\%) & SPAN 80 (\%) \\
\hline 1 & 94,75 & 5 & 0,25 \\
2 & 89,75 & 10 & 0,25 \\
3 & 79,75 & 20 & 0,25 \\
4 & 69,75 & 30 & 0,25 \\
5 & 59,75 & 40 & 0,25 \\
6 & 49,75 & 50 & 0,25 \\
7 & 39,75 & 60 & 0,25 \\
8 & 29,75 & 70 & 0,25 \\
& & &
\end{tabular}

Todos os testes foram realizados em duplicata. Os resultados foram organizados em gráficos para melhor visualização.

\section{RESULTADOS E DISCUSSÃO}

Para o preparo de $200 \mathrm{ml}$ de emulsão foram utilizados $0,25 \%$ de SPAN 80, em volume. $\mathrm{O}$ volume de óleo diesel, equivalente a proporção de cada emulsão $\mathrm{A} / \mathrm{O}$, foi misturada ao SPAN 80 em um béquer, e agitada no homogeneizador mecânico à 8000 RPM por 5 minutos, a proporção equivalente de água destilada foi adicionada aos poucos durante a agitação.

Foi observada a estabilidade da emulsão avaliando o tempo de separação das fases por influência gravitacional. Segundo a classificação de Santana (2013), todas as emulsões apresentaram estabilidade forte, pois levaram mais que uma hora para que houvesse a separação das fases.

Quanto ao comportamento reológico todas as emulsões apresentaram comportamento Newtoniano, como pode ser observado na Figura 3. 
Figura 3 - Curva de Fluxo para cada emulsão.

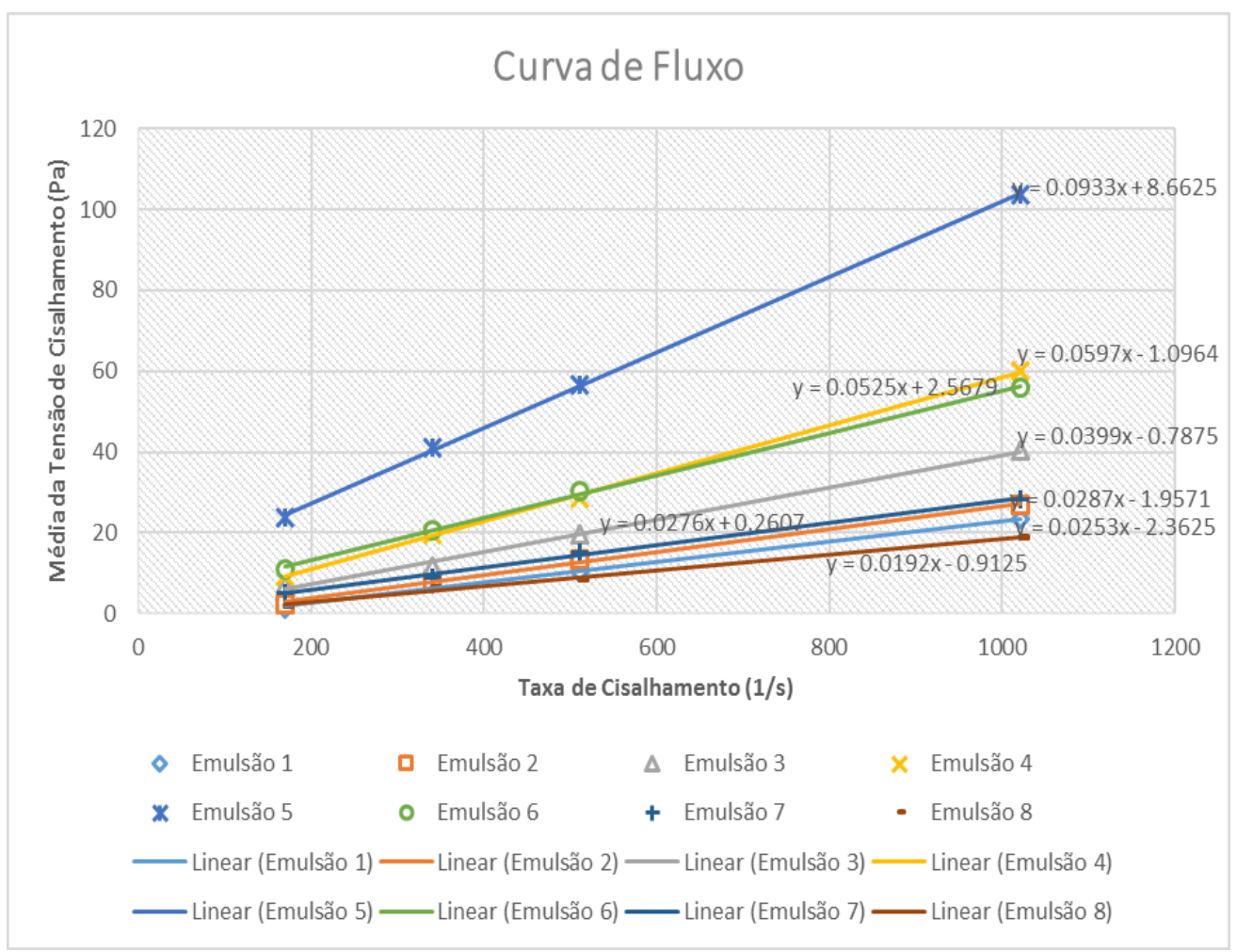

Ao lado de cada curva de tendência foi plotada com o auxílio do software Excel, a equação da curva, para o modelo linear, e foi comparado ao modelo Newtoniano para determinar a viscosidade da emulsão, os resultados da viscosidade são apresentados na Tabela 2. A Figura 4 apresenta os valores de viscosidade medidos pelo viscosímetro OFITE MODEL 900 à 600 RPM (rotações por minutos).

Figura 4 - Curva de viscosidade média em função do teor de água de cada emulsão medida à 600 RPM no viscosímetro.

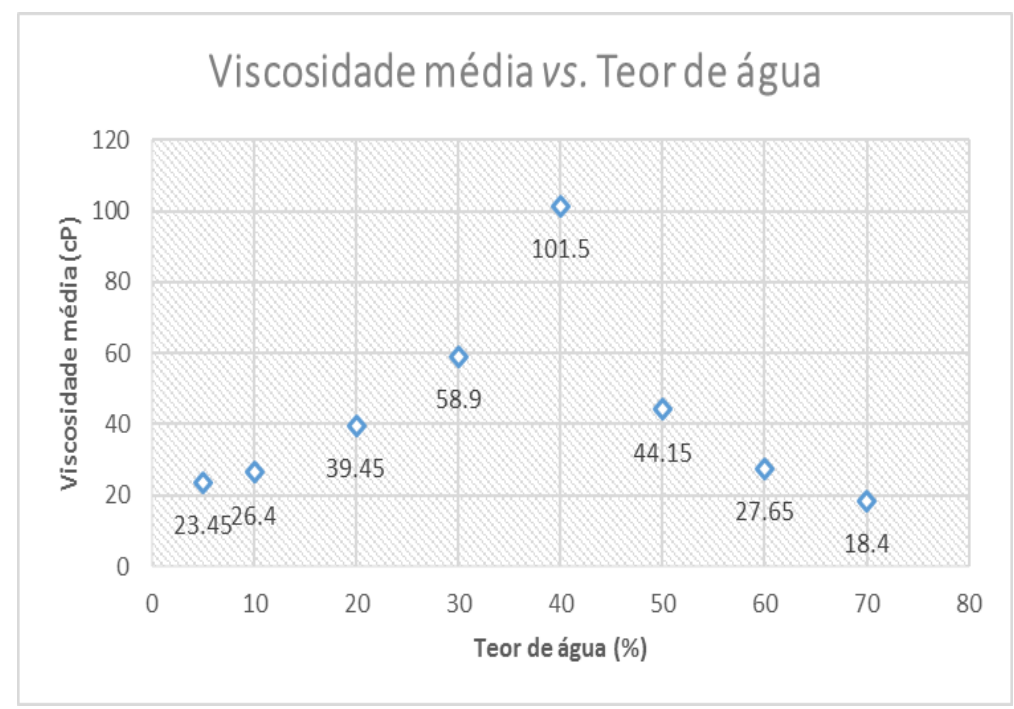


A Tabela 2 reúne os valores de viscosidade calculado através da curva de fluxo e através da viscosidade medida pelo viscosímetro. Esta tabela tem por finalidade comparar os valores calculados e experimentais da viscosidade média das amostras. Para formulação da equação foram utilizados os valores de tensão e taxa de cisalhamento, Pa (Pascal) e $\mathrm{s}^{-1}$ (1/segundos), respectivamente. O que gerou um termo para viscosidade média com unidade de Pa.s. Para fins de comparação a unidade da viscosidade média foi transformada de Pa.s para cP (centipoise).

Tabela 2 - Análise comparativa dos valores médios de viscosidade, calculado por meio de equação versus o valor experimental

\begin{tabular}{c|ccc} 
Emulsão & Equação $y=f(x)$ & $\begin{array}{c}\text { Viscosidade média das } \\
\text { amostras pela equaçao } \\
\text { do gráfico }(c P)\end{array}$ & $\begin{array}{c}\text { Viscosidade média das } \\
\text { amostras pelo OFITE }(c P) \grave{a}\end{array}$ \\
\hline 1 & $0,0253 \mathrm{x}-2,3625$ & 25,3 & $600 R P M$ \\
2 & $0,0287 \mathrm{x}-1,9571$ & 28,7 & 23,45 \\
3 & $0,0399 \mathrm{x}-0,7875$ & 39,9 & 26,4 \\
4 & $0,0597 \mathrm{x}-1,0964$ & 59,7 & 39,45 \\
5 & $0,0933 \mathrm{x}+8,6625$ & 93,3 & 58,9 \\
6 & $0,0525 \mathrm{x}+2,5679$ & 52,5 & 101,5 \\
7 & $0,0276 \mathrm{x}+0,2607$ & 27,6 & 44,15 \\
8 & $0,0192 \mathrm{x}-0,9125$ & 19,2 & 27,65 \\
\end{tabular}

Analisando separadamente o valor da viscosidade média para cada emulsão, observamos que os valores medidos e calculados são suficientemente próximos. Podemos então utilizar os valores calculados para determinar a característica da viscosidade das emulsões.

\section{CONCLUSÃO}

O entendimento do comportamento das emulsões é de grande importância, pois a determinação das suas características corrobora para uma melhor eficiência na escolha do método de separação das emulsões em campos de petróleo.

A emulsões formadas por água e óleo diesel para este experimento tem uma estabilidade forte. Portanto, mesmo que a viscosidade do óleo diesel seja diferente das viscosidades encontradas nos campos de petróleo, as emulsões formadas são fortes e de difícil separação.

Observando o comportamento do fluido, percebemos o seu caráter Newtoniano, Figura 3 , à medida que foi aumentada a taxa de cisalhamento ocorreu o aumento na viscosidade. 
Os experimentos mostraram que o valor da viscosidade da emulsão também aumenta com o aumento da proporção de água. Este crescimento gradual na viscosidade ocorre até que se atinja um ponto máximo. Este ponto representa o limite da fração volumétrica de água onde ocorre a inversão da emulsão. A importância no conhecimento do ponto de inversão da emulsão é fundamental na indústria do Petróleo devido a diminuição no valor da viscosidade durante procedimentos de escoamento.

\section{REFERÊNCIAS}

HENRÍQUEZ, C. J. M. W/O Emulsions: Formulation, Characterization and Destabilization. Caracas. Venezuela. 2009.

HERMANY, L. Aproximações estabilizadas de escoamento de fluidos viscoplásticos através de uma expansão seguida de uma contração axissimétrica. Programa de pósgraduação em Engenharia Mecânica. Universidade Federal do Rio Grande do Sul. Porto Alegre. 2012.

LAKE, L. W. Petroleum Engineering Handbook - General Engineering. Vol. I. Cap 12. Society of Petroleum Engineering (SPE). 2006.

NASCIMENTO, C. R. Reologia e Reometria Aplicadas ao Estudo de Polpas Minerais. Centro de Tecnologia Mineral - CETEM. Rio de Janeiro. 2008.

PEREIRA, L. J. B.; GARCIA-ROJAS, E. E. Emulsões múltiplas: formação e aplicação em microencapsulamento de componentes bioativos. Ciência Rural. Santa Maria, v. 45, n.1. p 155-162. Jan. 2015.

SANTANA, R. de C.; Surfactantes Self- Assembly. Aula 2. Tópicos Especiais em Engenharia de Produção. Departamento de Engenharias e Tecnologia. Universidade Federal do Espírito Santo. 2013.

SOUZA, T. A.; Inversão de emulsões de petróleo usando partículas sólidas. Programa de Pós Graduação em Engenharia Química. Universidade Federal do Paraná. Curitiba. 2009 